\title{
Non-analytic microscopic phase transitions and temperature oscillations in the microcanonical ensemble: An exactly solvable $1 d$-model for evaporation
}

\author{
Stefan Hilbert* \\ Institute for Physics, Humboldt-Universität zu Berlin, Newton-Straße 15, D-12489 Berlin, Germany \\ Jörn Dunke $\oplus$ \\ Institute for Physics, Universität Augsburg, Universitätsstraße 1, D-86135 Augsburg, Germany
}

(Dated: May 13, 2019)

\begin{abstract}
We calculate exactly both the microcanonical and canonical thermodynamic functions (TDFs) for a one-dimensional model system with piecewise constant Lennard-Jones type pair interactions. In the case of an isolated $N$-particle system, the microcanonical TDFs exhibit $(N-1)$ singular (non-analytic) microscopic phase transitions of the formal order $N / 2$, separating $N$ energetically different evaporation (dissociation) states. In a suitably designed evaporation experiment, these types of phase transitions should manifest themselves in the form of pressure and temperature oscillations, indicating cooling by evaporation. In the presence of a heat bath (thermostat), such oscillations are absent, but the canonical heat capacity shows a characteristic peak, indicating the temperature-induced dissociation of the one-dimensional chain. The distribution of complex zeros (DOZ) of the canonical partition may be used to identify different degrees of dissociation in the canonical ensemble.
\end{abstract}

PACS numbers: 05.70.Ce, 05.70.Fh, 36.40.Ei, 64.60.Cn

\section{INTRODUCTION}

Many macroscopic systems exhibit sudden variations of their physical properties (elasticity, conductivity, etc.), when one or more external control parameters (e.g., energy $E$, temperature $T$ ) pass certain critical values $[1,2]$. Such phenomena are usually referred to as phase transitions (PTs). Seminal contributions to the theory of PTs are due to, e.g., Mayer et al. 3, 4, 5], Onsager [6], van Hove 7], Yang and Lee 8, 9], Fisher [10], Grossmann et al. 11, 12, 13], Burkhardt 14], Pettini et al. 15, 16, 17] and Cuesta and Sánchez 18. These authors have studied in detail canonical ensembles (CEs) and grandcanonical ensembles, corresponding to systems in contact with heat bath and particle reservoirs.

Singular, or equivalently non-analytic, PTs are indicated by a discontinuity in the thermodynamic functions (TDFs) or one of their derivatives [19]. Sometimes it is also useful to consider smooth PTs, characterized by a strong but analytic variation in the TDFs 20, 21, 22, 23]. In the presence of a heat bath, singular canonical PTs can occur in the thermodynamic limit only 8, 9, 10, 11, 12, 13], whereas finite canonical systems may, at best, exhibit smooth PTs 20, 21, 22, 23]. However, the situation changes if the system under consideration is thermally isolated, corresponding to a microcanonical ensemble (MCE). Due to the different physical conditions underlying $\mathrm{MCE}$ and $\mathrm{CE}$, respectively, one can obtain significantly different predictions for several observable quantities 24, 25, 26, 27, 28, 29]. For example, in certain cases, microcanonical heat capacities can also be negative (e.g. in self-gravitating systems) whereas canonical heat capacities are generally positive. In particular, as will also be shown below, the microcanonical TDFs of finite isolated systems may exhibit non-analyticities. These singularities reflect evaporation/dissociation phenomena and may be interpreted as microscopic PTs in the small system [30].

The main objective of this article is to exemplify the differences between the MCE and $\mathrm{CE}$ and to elucidate particular observational consequences by means of a $1 d$ model for evaporation. Remarkably, this model system analyzed below allows for calculating exactly both the canonical and microcanonical TDFs for an arbitrary number of particles. The knowledge of the exact TDFs for both ensembles provides the basis for a detailed comparison of observables. Our main results can be summarized as follows:

In the case of the MCE the model system exhibits $(N-1)$ singular microscopic PTs, reflected by nonanalytic kinks in the caloric curve $T(E)$ and the pressure curve $P(E)$ at certain critical energy values $E_{k}$. The values $E_{k}$ can be identified with the binding energy of different dissociation states; i.e., the singularities (non-analyticities) separate energetically different evaporation phases. These microscopic PTs are accompanied by strong temperature oscillations; i.e., the temperature of the system decreases when increasing the energy in the vicinity of the critical values $E_{k}$. This effect corresponds to cooling by evaporation (or dissociation). By contrast, non-analytic transitions are absent in the corresponding CE; i.e., if the system is embedded into a heat bath of temperature $T$. Nevertheless, a smooth PT is observed that also persists in the thermodynamic limit even though the existence of a singular macroscopic PT is excluded by the (generalized) van Hove theorem [7, 18. Finally, our study of the distribution of complex zeros 
(DOZ) of the canonical partition function 20, 21, 22, 23] suggests that the DOZ may be used to identify different degrees of dissociation in the $\mathrm{CE}$.

\section{THE MODEL}

We consider a one-dimensional model system corresponding to $N$ identical point particles confined by a one-dimensional box of size $L$. The Hamiltonian reads

$$
H(\mathbf{p}, \mathbf{q} ; L, N)=\frac{\mathbf{p}^{2}}{2 m}+U(\mathbf{q} ; L, N)=E,
$$

with $\mathbf{q}=\left(q_{1}, \ldots, q_{N}\right)$ denoting the coordinates and $\mathbf{p}=\left(p_{1}, \ldots, p_{N}\right)$ the conjugate momenta. In the case of an isolated system the total energy $E$ is conserved. The potential energy $U=U_{\text {int }}+U_{\text {box }}$ is determined by the interaction potential

$$
U_{\mathrm{int}}(\mathbf{q} ; N)=\frac{1}{2} \sum_{\substack{i, j=1 \\ i \neq j}}^{N} U_{\text {pair }}\left(\left|q_{i}-q_{j}\right|\right)
$$

and the box potential

$$
U_{\text {box }}(\mathbf{q} ; L, N)= \begin{cases}0, & \mathbf{q} \in[0, L]^{N}, \\ +\infty, & \text { otherwise. }\end{cases}
$$

The pair potential is given by

$$
U_{\text {pair }}(r)= \begin{cases}\infty, & r \leq d_{\mathrm{hc}}, \\ -U_{0}, & d_{\mathrm{hc}}<r<d_{\mathrm{hc}}+r_{0}, \\ 0, & r \geq d_{\mathrm{hc}}+r_{0},\end{cases}
$$

where $d_{\mathrm{hc}}>0$ is the hard-core diameter of a particle with respect to pair interactions. The interaction potential (2C) can be viewed as a simplified Lennard-Jones potential. The depth of the potential well is determined by the binding energy parameter $U_{0}>0$ and the interaction range by the parameter $r_{0}$, where we shall additionally impose that

$$
0<r_{0} \leq d_{\mathrm{hc}}
$$

The latter condition ensures that particles may interact with their nearest neighbors only. Furthermore, we assume that $L>L_{\min } \equiv(N-1)\left(d_{\mathrm{hc}}+r_{0}\right)$, i.e., the volume is assumed to be sufficiently large for realizing the completely dissociated state, corresponding to $U=0$. The energy $E$ of the system can take values between the ground state energy

$$
E_{0} \equiv-(N-1) U_{0}
$$

and infinity.

\section{MICROCANONICAL ENSEMBLE}

The microcanonical ensemble (MCE) refers to an isolated system. Thence, the control parameters are energy $E$, volume $L$ and particle number $N$. The thermodynamic (Hertz) entropy of the MCE is given by $30,31,32,33$.

$$
S(E, L, N)=k_{B} \ln \Omega(E, L, N),
$$

where $k_{B}$ is the Boltzmann constant, and

$$
\Omega(E, L, N)=\frac{1}{N ! h^{N}} \int_{\mathbb{R}^{N}} \mathrm{~d} \mathbf{q} \int_{\mathbb{R}^{N}} \mathrm{~d} \mathbf{p} \Theta(E-H)
$$

the phase volume ( $h$ is Planck's constant, and $\Theta(x) \equiv 0$ for $x<0$ and $\Theta(x) \equiv 1$ for $x \geq 0)$. Using $N$-dimensional spherical momentum coordinates, one can rewrite Eq. (3b) as

$$
\begin{aligned}
\Omega & =C(N) \int_{\mathbb{R}^{N}} \mathrm{~d} \mathbf{q}(E-U)^{N / 2} \Theta(E-U), \\
C(N) & \equiv \frac{2(2 \pi m)^{N / 2}}{\Gamma(N / 2) N ! N h^{N}}
\end{aligned}
$$

where $\Gamma$ denotes the Euler gamma function. For Hamiltonian (11) one can calculate integral (4a) exactly, yielding (see Appendix)

$$
\Omega=C \sum_{k=0}^{N-1} \omega_{k}\left(E+k U_{0}\right)^{N / 2} \Theta\left(E+k U_{0}\right),
$$

where, for $L>(N-1)\left(r_{0}+d_{\mathrm{hc}}\right)$,

$$
\begin{aligned}
& \omega_{k}(N, L)=\left(\begin{array}{c}
N-1 \\
k
\end{array}\right) \sum_{i=0}^{k}\left(\begin{array}{l}
k \\
i
\end{array}\right)(-1)^{i} \times \\
& \times\left[L-(N-1) d_{\mathrm{hc}}-r_{0}(N-1-k+i)\right]^{N} .
\end{aligned}
$$

Given Eqs. (5), the microcanonical temperature $T$ and pressure $P$ are obtained from the standard definitions $T^{-1} \equiv \partial S / \partial E$ and $P / T \equiv \partial S / \partial L[2,33,34,35]$. For example, for the temperature one finds

$$
k_{B} T=\frac{2}{N} \frac{\sum_{k=0}^{N-1} \omega_{k}\left(E+k U_{0}\right)^{N / 2} \Theta\left(E+k U_{0}\right)}{\sum_{k=0}^{N-1} \omega_{k}\left(E+k U_{0}\right)^{N / 2-1} \Theta\left(E+k U_{0}\right)},
$$

which reduces to the ideal gas law $E=N k_{B} T / 2$ in the limit $E \gg N U_{0}$. It is worthwhile to recall that, for a Hamiltonian of the form (11), the thermal energy (6) derived from the Hertz entropy is directly related to the microcanonical mean kinetic energy per degree of freedom by virtue of the equipartition theorem [33, 34]:

$$
\frac{k_{B} T}{2}=\left\langle\frac{p_{i}^{2}}{2 m}\right\rangle, \quad i=1, \ldots, N
$$


where $\langle\cdot\rangle$ denotes the average with respect to the microcanonical probability density function

$$
f(q, p)=\left(\frac{\partial \Omega}{\partial E}\right)^{-1} \frac{1}{N ! h^{N}} \delta(E-H(q, p)) .
$$

Hence, for isolated ergodic systems with an arbitrary particle number $N$, the caloric law $T(E)$ can be measured experimentally by monitoring the kinetic energy over a sufficiently long time interval (at fixed energy values $E$ ).

As shown in Fig. 1] the microcanonical caloric law (6) as well as the pressure $P(E)$ exhibit $N$ non-analytic points at the energies $E_{k}=-k U_{0}, k=0, \ldots, N-1$, separating $N$ energetically different dissociation states (all bindings intact, one binding broken, etc.). The formal order 30, 36, 37] of these non-analyticities equals $N / 2$, i.e., the entropy has continuous derivatives up to order $(N / 2-1)$, but the $(N / 2)$ th derivative becomes discontinuous (a similar result was obtained recently by Kastner and Schnetz for the mean-field spherical spin model [38]; see also Gross 39] for a general discussion of differentiability properties of the microcanonical partition function). Consequently, the "microscopic (dissociation) phases' as well as the singularities appear to be smoothened out in the thermodynamic limit $N \rightarrow \infty$. Nevertheless, for finite systems - and in particular at small densities - the non-analytic behavior is accompanied by strong variations/oscillations of observable quantities as temperature and pressure, when continuously varying $E$. Both qualitatively and quantitatively, this behavior is analogous to what is usually denoted as a 'phase transition'. However, since these microscopic nonanalyticities do not survive in the thermodynamic limit (at least for our $1 d$-model), they strictly speaking are not covered by the conventional definition of singular macroscopic PTs. We shall, therefore, speak of singular (or non-analytic) microscopic PTs in the MCE [48].

Let us also briefly discuss the parameter dependence of the microcanonical TDFs shown in Fig. 1 As evident from Eq. (5a), the positions $E_{k}$ of the singular macroscopic PTs are just proportional to $U_{0}$. The amplitude of the associated oscillations in $T$ and $P$ does also depend on the particle number $N$ and box size $L$ : The strength of the oscillations increases for larger values $U_{0}$ and $L$, but becomes smaller for larger particle numbers $N$. The number and formal order of the PTs, however, only depend on the particle number $N$ and are independent of $U_{0}$ and $L$ (as long as $U_{0}>0$ and $L>L_{\min }$ ). Thus, qualitatively, the results are independent of the particular choice of the model parameters $U_{0}$ and $L$. Moreover, analogous features can be found in the microcanonical caloric curves of $1 d$ Lennard-Jones chains [30].

It should be mentioned that the exact phase volume (5a) of our model system resembles in structure the phase volume obtained by the Harmonic Superposition Method (HSM) applied to Lennard-Jones clusters (see, e.g., Doye [40] or Wales and Doye 25] and references therein). The HSM approximates the phase volume $\Omega(E)$ by a sum of ellipsoidal regions around all local minima of the potential $U$ lower than the total energy $E$. This method has been successfully applied to describe melting phenomena, as e.g. the low-temperature properties of $3 d$ Lennard-Jones clusters and their transition from a solid-like state, where the cluster only vibrates around the ground state configuration, to a liquid-like state, where also other locally stable configurations energetically accessible. The standard HSM approximation, however, does not properly account for the contribution to the phase volume stemming from (partly) dissociated (or gas) states of the cluster, and therefore, is not suitable for describing evaporation phenomena. In particular, the HSM does not yield any singular microscopic PTs for $1 d$ Lennard-Jones chains (where only one locally stable configuration, i.e. the ground state, exists) therewith contradicting exact analytical and numerical results [30]. By contrast, the model system discussed here - if considered as an approximation to Lennard-Jones chains - does reproduce these microscopic PTs related to evaporation (but, of course, our model cannot be applied to melting processes because it is one-dimensional).

It is worthwhile to discuss the microscopic PTs and the origin of the associated temperature oscillations, as observed in our model, from a more general point of view. Mathematically, microscopic PTs of the above type arise whenever the phase volume $\Omega$ grows non-smoothly in the vicinity of some critical energy value $E=E_{k}$. This can best be illustrated by considering the energetically admissible subset of the configuration space

$$
\mathcal{A}(E)=\left\{q \in \mathbb{R}^{N} \mid \Theta(E-U(q))=1\right\} .
$$

The set $\mathcal{A}(E)$ consists of all positions space points $q=$ $\left(q_{1}, \ldots, q_{N}\right)$ that can be occupied by the system at the given energy value $E$. Clearly, the boundary of $\mathcal{A}$, denoted by $\partial \mathcal{A}$, determines the effective range of the integral in Eq. 4a . Hence, whenever $\mathcal{A}$ or $\partial \mathcal{A}$, respectively, change their shape in an irregular (non-analytic) manner, a non-analyticity in the phase volume $\Omega$ may arise (and, hence, in the TDFs). For example, such a irregular change in the shape of $\mathcal{A}$ occurs when the energy for the next dissociation step is crossed, since then some parts of the boundary $\partial \mathcal{A}$ suddenly become determined by the box potential.

It remains to be discussed how the temperature oscillations - i.e., the regions with negative heat capacity (also known as 'S-bends' or van der Waals-type loops [49]) arise: In the vicinity of the dissociation energy $E_{k}$, the set $\mathcal{A}$ and, thus, also $\Omega$ and $S$ grow very rapidly, thereby giving rise to a drop-off in temperature. Geometrically, this can be viewed as a sudden increase of the 'effective dimensionality' of $\mathcal{A}$. Here, 'effective dimensionality' refers to the number of orthogonal configuration space directions in which $\mathcal{A}$ has an extent comparable to the system 
size $L$. Hence, typically, the temperature oscillations appear more pronounced for larger values of $L$. From the physical point of view, the temperature decrease after the $k$ th dissociation step just means that for energy values slightly larger than $E_{k}$ the dissociated fragments have very little kinetic energy (since most of the energy has already been used to break the binding). The larger the system the less likely it is that the fragments temporarily recombine in a state of high kinetic energy; i.e., from a probabilistic standpoint, the average (17) is then dominated by phase space regions of low kinetic energy. With regard to practical applications, this means that one could cool such a small isolated system of bound particles by injecting energy until the fragmentation process sets in (cooling by evaporation/dissociation).

The above described features of microscopic PTs are generic and shared by all physical systems that exhibit dissociation and evaporation (e.g. similar microscopic PTs and temperature oscillations are also found for small $1 d$-Lennard-Jones molecules [30]). In particular, microscopic PTs should become more pronounced in two or three dimensions (since then effective dimension of $\mathcal{A}$ grows even more rapidly at the dissociation levels) and also be observable in quantum systems. By virtue of dissociation experiments with small particle numbers and very low densities (similar to those of Schmidt et al. [41], but without heat bath), one should, in principle, be able to detect the oscillating behavior e.g. in temperature and pressure curves. However, to actually observe such oscillations one has to realize the requirements of the $\mathrm{MCE}$, i.e. a thermally isolated system with regulated energy injection. Furthermore, due to the microscopic origin of the oscillations and the requirement of a relatively low particle density, a high sensitivity of the velocity and force measurements and long measuring time spans will be necessary.

\section{CANONICAL ENSEMBLE}

Employing the canonical ensemble (CE) is appropriate, if the system under consideration is in thermal contact with a much larger system (heat bath), as e.g. realized in dusty cluster experiments 42 . The relevant thermodynamic potential is the free energy [2]

$$
F(\beta, L, N) \equiv-\beta^{-1} \ln z_{\mathrm{C}}(\beta, L, N)
$$

where $z_{\mathrm{C}}$ is the canonical partition function. The external control variables are now the inverse temperature $\beta \equiv\left(k_{B} T\right)^{-1}$ of the heat bath, the volume $L$ and the particle number $N$. For the above model, $z_{\mathrm{C}}$ can be exactly calculated, analogous to Eq. (15), as

$$
z_{\mathrm{C}}=\frac{1}{N !}\left(\frac{2 \pi m}{\beta h^{2}}\right)^{N / 2} \sum_{k=0}^{N-1} \omega_{k} e^{\beta k U_{0}}
$$
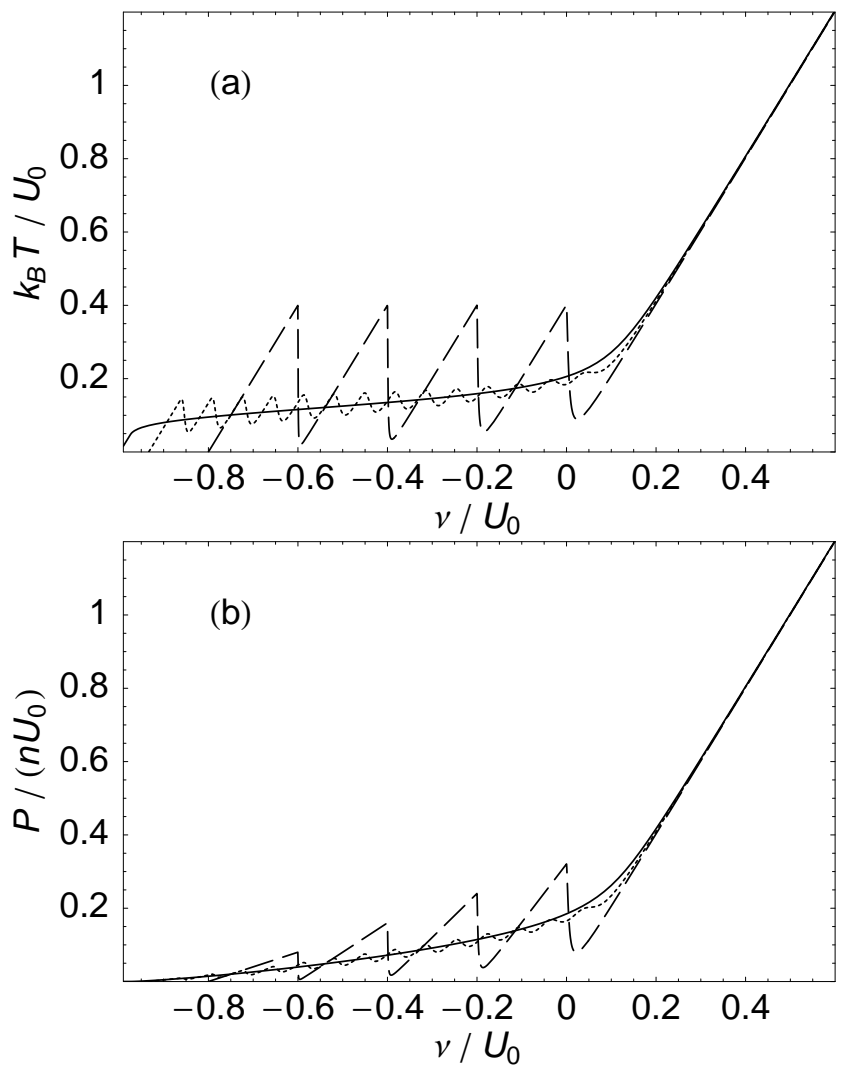

FIG. 1: (a) Microcanonical temperature $T$ and (b) pressure $P$ as a function of energy per particle $\nu=E / N$ for a (reduced) density $n=N /\left[L-(N-1) d_{\mathrm{hc}}\right]=0.001 / r_{0}$ and different number of particles $N=5$ (dashed line), $N=15$ (dotted), and $N=500$ (solid). Note that each of the curves is $(N / 2-2)$ times differentiable.

with $\omega_{k}(N, L)$ given by Eq. (5b). Mean energy and pressure of the $\mathrm{CE}$ are defined by $\bar{E} \equiv-\partial\left(\ln z_{\mathrm{C}}\right) / \partial \beta$ and $\bar{P} \equiv-\partial F / \partial L$, yielding e.g. the canonical caloric law

$$
\bar{E}=\frac{N}{2 \beta}-\frac{\sum_{k=0}^{N-1} \omega_{k} e^{\beta k U_{0}} k U_{0}}{\sum_{k=0}^{N-1} \omega_{k} e^{\beta k U_{0}}} .
$$

Figures 2 (a) and (b) show $\bar{E}(T)$ and $\bar{P}(T)$ for different values of the reduced particle density $n=N /\left[L-(N-1) d_{\mathrm{hc}}\right]$. In contrast to the microcanonical pressure [Fig. [1 (b)], the canonical pressure is a monotonous function of $T$ or $\bar{E}$, respectively. In the thermodynamic limit, microcanonical and canonical caloric curves become nearly indistinguishable. The canonical heat capacity $\bar{c}_{L}=\partial \bar{E} / \partial T$ exhibits a strong (nonsingular) peak in the temperature region, where dissociation occurs [Fig. 2](c)]. If observed in an experimentally measured curve, such behavior would possibly be interpreted as a PT. For decreasing density $n$, the position of the maximum of $\bar{c}_{L}$ moves closer to $T=0$, while its height increases rapidly. Furthermore, our results indicate that for $N \geq 15$ the TDFs $\bar{\nu}$ and $\bar{c}_{L}$ become virtually independent of $N$. The (non-singular) peak in the heat 

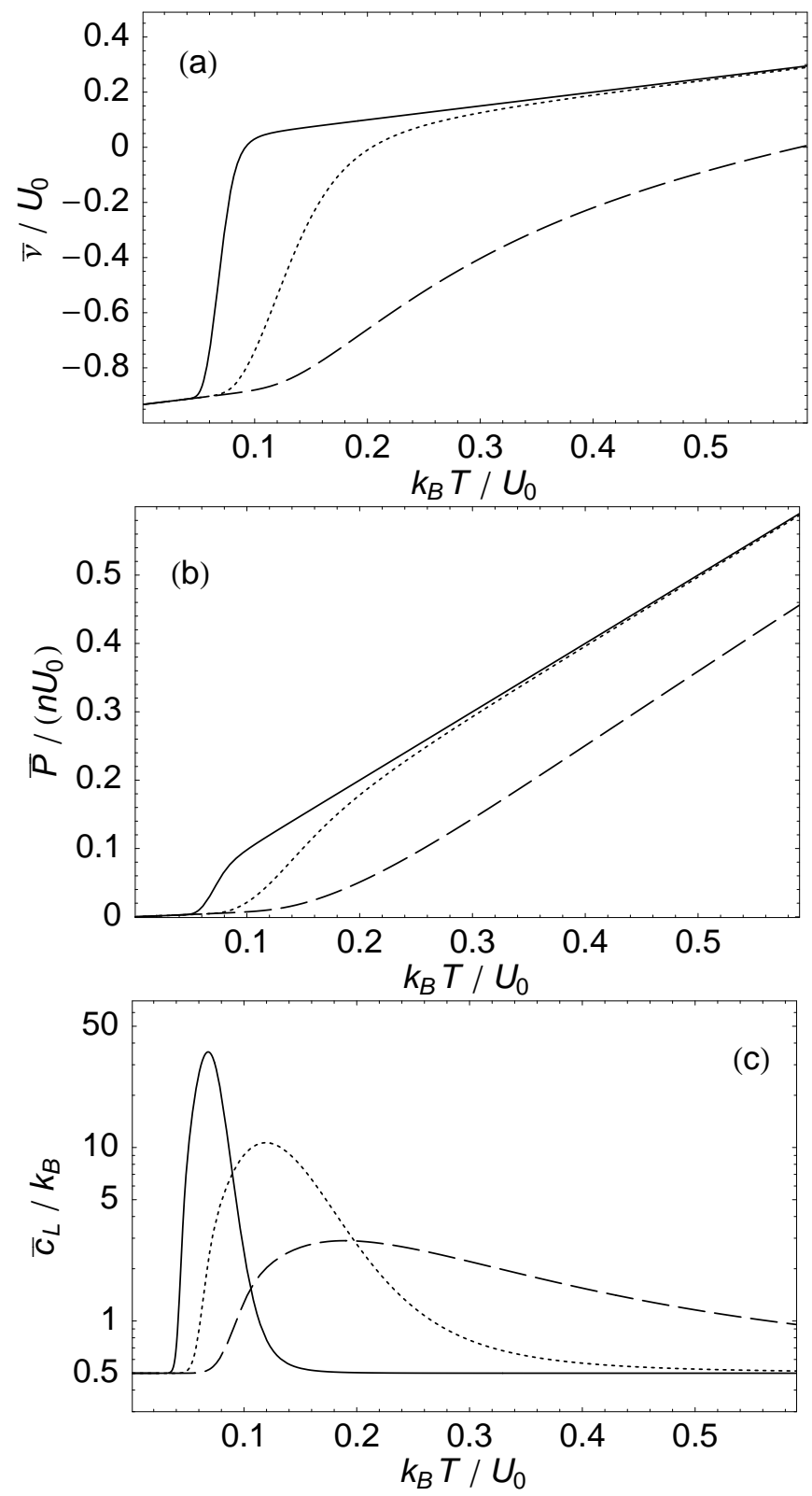

FIG. 2: (a) Canonical mean energy per particle $\bar{\nu}=\bar{E} / N$, (b) pressure $\bar{P}$ and (c) specific heat capacity $\bar{c}_{L}=\partial \bar{E} / \partial T$ (logarithmic scale) as a function of temperature $T$ for $N=15$ particles and different values of the reduced density $n=10^{-1} / r_{0}$ (dashed line), $n=10^{-3} / r_{0}$ (dotted), and $n=10^{-6} / r_{0}$ (full).

capacity persists in the thermodynamic limit (analogous to the $1 d$ Ising model [43]).

To obtain a more detailed characterization of the dissociation process in the $\mathrm{CE}$, we next study the distribution of complex zeros $(\mathrm{DOZ})$ of $z_{\mathrm{C}}$. As evident from Eq. (11), the only relevant configurational part of $z_{\mathrm{C}}(\beta)$ is a polynomial of degree $(N-1)$ in $z=e^{\beta U_{0}}$, and, therefore, has $(N-1)$ complex Fisher zeros 10 per branch of the logarithm. This quasi-polynomial structure is a consequence of the fact that, for our specific model, the configuration space $[0, L]^{N}$ can be partitioned into regions of equal total binding energy $E_{k}=-k U_{0}, k=0, \ldots, N-1$ (see Appendix). Since all zeros can be obtained by adding integer multiples of $2 \pi i$ to the zeros $\beta_{k}$ of the main branch (for which $\Im\left(\beta_{k}\right)=\pi$ ), it suffices to discuss to the main branch only while bearing in mind that each zero $\beta_{k}$ in the main branch is associated with an infinite set of zeros $\left\{\beta_{k}+2 \pi i s \mid s \in \mathbb{Z}\right\}$. Ordering the zeros according to their real parts, $\Re\left(\beta_{0}\right) \leq \ldots \leq \Re\left(\beta_{N-1}\right)$, we find that the region of the $\bar{c}_{L}$-peak is well-described by the temperature interval $\left[\Re\left(\beta_{N-1}\right)^{-1}, \Re\left(\beta_{0}\right)^{-1}\right]$.

The asymptotic behavior of the DOZ for $N \rightarrow \infty$ may be used to characterize the parameters (critical temperature, order, etc.) of singular macroscopic PTs 8, $9,10,11,12,13$. In our model, the Fisher zeros are located at least a distance $\pi$ away from the the real $\beta$ axis regardless of particle number $N$ (see Fig. 3). Hence, the zeros cannot converge to a point on the real $\beta$-axis. The peculiar position of the zeros thus ensures agreement with the (generalized) van Hove theorem 7, 18] which excludes the existence of a singular macroscopic PT in our model.

The DOZ has also been employed to study their finite size analogs of macroscopic PTs 20, 21, 22, 23]. Applying these methods to our model, one may interpret the smooth phase transition observed in the $\mathrm{CE}$ as a superposition of $(N-1)$ smooth microscopic 'first-order' phase transitions indicated by the $(N-1)$ sets of zeros $\left\{\beta_{k}+\right.$ $2 \pi i s \mid s \in \mathbb{Z}\}$. One can then use $\left\{\Re\left(\beta_{0}\right), \ldots, \Re\left(\beta_{N-1}\right)\right\}$ to distinguish (define) different dissociation states in the CE. Thus, our results suggest that the DOZ encodes detailed information about the observed smooth phase transition and the energetically different degrees of dissociation even if there is no singular PT in the thermodynamic limit. In particular, since microcanonical phase volume and canonical partition function can be mapped onto each other via the Laplace transformation [44, 45], one may speculate that there exists a direct mathematical link between the singular microscopic PTs in the MCE and the DOZ in the CE.

\section{SUMMARY}

We have studied a simple $1 d$-evaporation model with nearest neighbor interaction potentials characterized by a hard-core repulsive part and piecewise constant shortrange attraction. By analyzing the exact TDFs of this model, it was shown that, in the case of a thermally isolated system (MCE), the microcanonical caloric and pressure laws exhibit singularities, separating different dissociation states. The formal order of these non-analyticities increases as the particle number increases. Hence, the microscopic PTs vanish in the thermodynamic limit and are intrinsically different from the topologically induced macroscopic PTs discussed in Refs. 15, 16, 17, 46, 47]. 


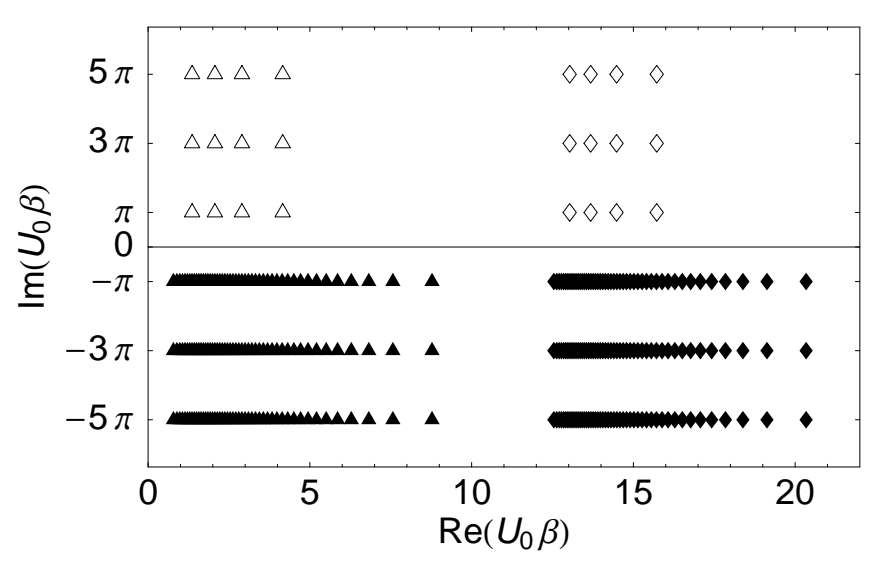

FIG. 3: DOZ of the canonical partition function $Z_{\mathrm{C}}(\beta)$ for $N=5$ (open symbols) and $N=15$ (filled symbols) and $n=10^{-1} / r_{0}$ (triangles), and $n=10^{-6} / r_{0}$ (diamonds). For better visibility, for $N=5$ (15) only the first few branches of zeros with positive (negative) imaginary part are shown (further branches can be obtained by shifting with multiples of $2 \pi i$ in the vertical direction; generally, the Fisher zeros are symmetric with respect to the real axis [20]).

For sufficiently low particle numbers and densities, the microscopic PTs are accompanied by strong oscillations of the temperature (mean kinetic energy) and pressure. These oscillations arise from a rapid change of the phase volume near the dissociation thresholds. They are a generic feature of particle systems with Lennard-Jones like interaction potentials [30, 39]. They are not restricted to one space dimension, but expected to be even stronger in two and three space dimensions, and should also be observable in quantum systems. In a suitably designed dissociation experiment, one should therefore, in principle, be able to detect the oscillating behavior e.g. in temperature and pressure curves. In particular, such temperature oscillations may provide the possibility to cool a small isolated system by means of regulated energy injection (cooling by evaporation).

If the model system is coupled to a heat bath (CE), a smooth PT is observed, but no singularities are found 7, 18]. Nevertheless, the DOZ seemingly permits to quantify the temperature range of the $\mathrm{PT}$ and to define different dissociation states.

We thus conclude this paper with two important questions, which need to be answered in the future: Is it possible to design an experiment that allows to observe singular microscopic phase transitions in finite size systems (or, at least, oscillatory behavior of thermodynamic observables such as pressure), as predicted by the microcanonical statistical theory? Can one find a direct mathematical link between singularities in microcanonical partition function of finite systems and the DOZ of the corresponding canonical partition function - and, thus, between microscopic and macroscopic phase transitions in arbitrary space dimensions?
The authors would like to thank M. Kastner, L. Velazquez-Abad and an anonymous referee for several helpful remarks.

* Electronic address: hilbert@mpa-garching.mpg.de

$\dagger$ Electronic address: joern.dunkel@physik.uni-augsburg.de

[1] L. P. Kadanoff, W. Götze, D. Hamblen, R. Hecht, E. A. S. Lewis, V. V. Palciauskas, M. Rayl, J. Swift, D. Aspnes, and J. Kane, Rev. Mod. Phys. 39, 395 (1967).

[2] L. D. Landau and E. M. Lifshitz, Statistical Physics, vol. 5 of Course of Theoretical Physics (ButterworthHeinemann, Oxford, 2003), 3rd ed.

[3] J. E. Mayer, J. Chem. Phys. 5, 67 (1937).

[4] J. E. Mayer and P. G. Ackermann, J. Chem. Phys. 5, 74 (1937).

[5] S. F. Streeter and J. E. Mayer, J. Chem. Phys. 7, 1025 (1939).

[6] L. Onsager, Phys. Rev. 65, 117 (1944).

[7] L. van Hove, Physica (Amsterdam) 16, 137 (1950).

[8] C. N. Yang and T. D. Lee, Phys. Rev. 87, 404 (1952).

[9] T. D. Lee and C. N. Yang, Phys. Rev. 87, 410 (1952).

[10] M. E. Fisher, in Critical Phenomena: Proceedings of the 51st Enrico Fermi Summer School, Varenna, Italy, edited by M. S. Green (Academic Press, New York, 1971), p. 1.

[11] S. Grossmann and W. Rosenhauer, Z. Phys. 207, 138 (1967).

[12] S. Grossmann and W. Rosenhauer, Z. Phys. 218, 437 (1969).

[13] S. Grossmann and V. Lehmann, Z. Phys. 218, 449 (1969).

[14] T. W. Burkhardt, J. Phys. A: Math. Gen. 14, L63 (1981).

[15] L. Casetti, E. G. D. Cohen, and M. Pettini, Phys. Rev. E 65, 036112 (2002).

[16] R. Franzosi and M. Pettini, Phys. Rev. Lett. 92, 060601 (2004).

[17] L. Angelani, L. Casetti, M. Pettini, G. Ruocco, and F. Zamponi, Phys. Rev. E 71, 036152 (2005).

[18] J. A. Cuesta and A. Sánchez, J. Stat. Phys. 115, 869 (2004).

[19] D. Ruelle, Statistical Mechanics (World Scientific, Singapore, 1999).

[20] P. Borrmann, O. Mülken, and J. Harting, Phys. Rev. Lett. 84, 3511 (2000).

[21] O. Mülken, H. Stamerjohanns, and P. Borrmann, Phys. Rev. E 64, 047105 (2001).

[22] W. Janke and R. Kenna, J. Stat. Phys 102, 1211 (2001).

[23] N. A. Alves, J. P. N. Ferrite, and U. H. E. Hansmann, Phys. Rev. E 65, 036110 (2002).

[24] D. J. Wales and R. S. Berry, Phys. Rev. Lett. 73, 2875 (1994).

[25] D. J. Wales and J. P. K. Doye, J. Chem. Phys. 103, 3061 (1995).

[26] D. H. E. Gross, Phys. Rep. 279, 119 (1997).

[27] P. Chomaz, V. Duflot, and F. Gulminelli, Phys. Rev. Lett. 85, 3587 (2000).

[28] D. H. E. Gross, Micro-canonical thermodynamics, vol. 66 of World Scientific Lecture Notes in Physics (World Scientific, Singapore, 2001).

[29] M. Costeniuc, R. S. Ellis, H. Touchette, and B. Turkington, J. Stat. Phys. 119, 1283 (2005). 
[30] J. Dunkel and S. Hilbert, Physica A (2005), in press (cond-mat/0511501).

[31] P. Hertz, Ann. Phys. (Leipzig) 33, 225 (1910).

[32] P. Hertz, Ann. Phys. (Leipzig) 33, 537 (1910).

[33] R. Becker, Theory of heat (Springer, New York, 1967).

[34] K. Huang, Statistical Mechanics (Wiley, NewYork, 1963).

[35] A. Münster, Statistical Thermodynamics, vol. 1 (Springer, Berlin, Heidelberg, New York, 1969).

[36] P. Ehrenfest and T. Ehrenfest, The conceptual foundation of the statistical approach in mechanics (Cornell University Press, Ithaca, NY, 1959), reprint of german 1912 edition.

[37] P. Ehrenfest, Proc. Amsterdam Acad. 36, 153 (1933).

[38] M. Kastner and O. Schnetz, J. Stat. Phys. 122, 1195 (2006).

[39] D. H. E. Gross (2004), cond-mat/0403582.

[40] J. P. K. Doye, Ph.D. thesis, Gonville \& Caius College, University of Cambridge, UK (1996).

[41] M. Schmidt, T. Hippler, J. Donges, W. Kronmüller, B. von Issendorff, H. Haberland, and P. Labastie, Phys. Rev. Lett. 87, 203402 (2001).

[42] O. S. Vaulina, S. V. Vladimirov, O. F. Petrov, and V. E. Fortov, Phys. Rev. Lett. 88, 245002 (2002).

[43] F. Schwabl, Statistische Mechanik (Springer, 2000), in german.

[44] E. M. Pearson, T. Halicioglu, and W. A. Tiller, Phys. Rev. A 32, 3030 (1985).

[45] O. Mülken, Ph.D. thesis, Universität Oldenburg, Germany (2001).

[46] M. Kastner, Phys. Rev. Lett. 93, 150601 (2004).

[47] A. C. R. Teixeira and D. A. Stariolo, Phys. Rev. E 70, 016113 (2004).

[48] Due to the fact that the formal Ehrenfest order of the microscopic PTs increases with particle number, it is not particularly helpful to classify microscopic PTs according to this order.

[49] Wales and Berry 24] and Wales and Doye 25] discuss necessary and sufficient criteria for the existence of Sbends in the microcanonical caloric curve based on the Boltzmann entropy, given by $S_{B}=k_{B} \ln (\partial \Omega / \partial E)$ with respect to our notation. However, in general, Eq. (7) does not hold for the temperature $T_{B}=\left(\partial S_{B} / \partial E\right)^{-1}$ derived from the Boltzmann entropy, which is why we prefer to use the Hertz entropy $S=k_{B} \ln \Omega$. The difference between Hertz entropy $S$ and Boltzmann entropy $S_{B}$ may become negligible in the thermodynamic limit, i.e., for sufficiently large systems, but is relevant for small systems.

\section{APPENDIX}

To calculate integral (4a), we first eliminate the hardcore part of the interaction potential by virtue of the transformation $L \mapsto \lambda=L-(N-1) d_{\mathrm{hc}}, \quad q_{i} \mapsto x_{i}=$ $q_{i}-n_{i} d_{\mathrm{hc}} \in[0, \lambda]$, here $n_{i}$ equals the number of particles $j$ with $q_{j}<q_{i}$. With these definitions the potential energy can be rewritten as:

$$
U^{\prime}(\mathbf{x} ; \lambda, N)=\frac{1}{2} \sum_{i, j}^{\prime} U_{\text {pair }}^{\prime}\left(\left|x_{i}-x_{j}\right|\right)+U_{\text {box }}(\mathbf{x} ; \lambda, N),
$$

where the sum $\sum^{\prime}$ goes over nearest neighbors only, and

$$
U_{\text {pair }}^{\prime}(r)= \begin{cases}-U_{0}, & 0<r<r_{0} \\ 0, & r \geq r_{0}\end{cases}
$$

For $k=0, \ldots, N-1$ the family of sets $\mathcal{G}_{k}=\left\{\mathbf{x} \in[0, \lambda]^{N} \mid U^{\prime}(\mathbf{x})=-k U_{0}\right\} \quad$ constitutes a partition (disjoint cover) of the configuration space permitted by the transformed box volume $[0, \lambda]$. Thus we can rewrite Eq. (4a) in the form

$$
\Omega=C(N) \sum_{k=0}^{N-1}\left(E+k U_{0}\right)^{N / 2} \Theta\left(E+k U_{0}\right) \operatorname{vol}\left(\mathcal{G}_{k}\right) .
$$

In order to calculate $\operatorname{vol}\left(\mathcal{G}_{k}\right)$ we first note that

$$
\omega_{k} \equiv \operatorname{vol}\left(\mathcal{G}_{k}\right)=N ! \cdot \operatorname{vol}\left(\mathcal{G}_{k}^{+}\right)
$$

where (writing $\left\{\mathbf{x} \in[0, \lambda]^{N} \mid \ldots\right\}=\{\ldots\}$ from now on)

$$
\mathcal{G}_{k}^{+}=\left\{x_{1}<x_{2}<\ldots<x_{N} \wedge U(\mathbf{q})=-k U_{0}\right\} .
$$

Next we note that

$$
\operatorname{vol}\left(\mathcal{G}_{k}^{+}\right)=\left(\begin{array}{c}
N-1 \\
k
\end{array}\right) \cdot \operatorname{vol}\left(\mathcal{G}_{k}^{++}\right)
$$

where

$$
\begin{aligned}
\mathcal{G}_{k}^{++}= & \left\{x_{1}<x_{2}<\ldots<x_{N}\right\} \cap \\
& \left\{\left(x_{2}-x_{1}<r_{0}\right) \wedge \ldots \wedge\left(x_{k+1}-x_{k}<r_{0}\right) \wedge\right. \\
& \left.\left(x_{k+2}-x_{k+1} \geq r_{0}\right) \wedge \ldots \wedge\left(x_{N}-x_{N-1} \geq r_{0}\right)\right\}, \\
\operatorname{vol}\left(\mathcal{G}_{k}^{++}\right)= & \int_{(N-1-k) r_{0}}^{\lambda} \mathrm{d} x_{N} \int_{(N-2-k) r_{0}}^{x_{N}-r_{0}} \mathrm{~d} x_{N-1} \times \ldots \\
& \int_{r_{0}}^{x_{k+3}-r_{0}} \mathrm{~d} x_{k+2} \int_{0}^{x_{k+2}-r_{0}} \mathrm{~d} x_{k+1} \times \\
& \int_{\left[x_{k+1}-r_{0}\right]_{+}}^{x_{k+1}} \mathrm{~d} x_{k} \cdots \int_{\left[x_{3}-r_{0}\right]_{+}}^{x_{3}} \mathrm{~d} x_{2} \int_{\left[x_{2}-r_{0}\right]_{+}}^{x_{2}} \mathrm{~d} x_{1} .
\end{aligned}
$$

The positive part $[x]_{+} \equiv \max \{0, x\}$ satisfies

$$
\begin{aligned}
\int \mathrm{d} x[x]_{+}^{n} & =\frac{[x]_{+}^{n+1}}{n+1}, & & n \in \mathbb{N} ; \\
{\left[[x-c]_{+}-c\right]_{+} } & =[x-2 c]_{+}, & & c>0 .
\end{aligned}
$$

By virtue of these identities, we can write Eq. (14) as

$$
\begin{aligned}
\operatorname{vol}\left(\mathcal{G}_{k}^{++}\right)= & \int_{(N-1-k) r_{0}}^{\lambda} \mathrm{d} x_{N} \int_{(N-2-k) r_{0}}^{x_{N}-r_{0}} \mathrm{~d} x_{N-1} \times \cdots \\
& \int_{r_{0}}^{x_{k+3}-r_{0}} \mathrm{~d} x_{k+2} \int_{0}^{x_{k+2}-r_{0}} \mathrm{~d} x_{k+1} K_{k},
\end{aligned}
$$

where

$$
K_{k}=\frac{1}{k !} \sum_{i=0}^{k}(-1)^{i}\left(\begin{array}{l}
k \\
i
\end{array}\right)\left[x_{k+1}-i r_{0}\right]_{+}^{k}
$$

Performing the remaining integrations and reversing the transformation $L \mapsto \lambda$, one obtains the final result (5b). 